\title{
Travelling fronts in an array of coupled symmetric bistable units
}

\author{
Diego Pazó ${ }^{1}$ and Vicente Pérez-Muñuzuri \\ Grupo de Física non Lineal, Facultade de Física, \\ Universidade de Santiago de Compostela, E-15782 Santiago de Compostela, Spain
}

\begin{abstract}
A symmetry breaking mechanism is shown to occur in an array composed of symmetric bistable Lorenz units coupled through a nearest neighbour scheme. When the coupling is increased, we observe the route: standing $\rightarrow$ oscillating $\rightarrow$ travelling front. In some circumstances, this route can be described in terms of a gluing of two cycles on the plane. In this case, the asymptotic behaviour of the velocity of the front is found straightforward. However, it may also happen that the gluing bifurcation involves a saddle-focus fixed point. If so, front dynamics may become quite complex displaying several oscillating and propagating regimes, including chaotic (Shil'nikov-type) front propagation. Finally, we compare our results with different couplings and with other discrete bistable media.
\end{abstract}

Key words: Travelling waves, Gluing bifurcation, Bistable equation, Homoclinic chaos PACS: 45.05.+x, 2.30.Oz, 5.45.Ac, 82.40.Ck

\section{Introduction}

Several areas of science use model equations of the reaction-diffusion type. Moreover, besides its special interest in some fields (chemistry [1,2], biology [3], ...) reaction-diffusion equations are considered as abstract models for pattern formation [4].

Real systems are frequently composed of discrete elements, material models and biological cells are just two examples. Therefore, it is natural to deal with the discrete space version of the reaction-diffusion equation. In this point, it must be emphasized that discreteness may manifest itself in a strong way, such that some phenomena are not simple analogies of the continuous ones.

$\overline{1 \text { Tel.: }}+34-981-547023$; fax: +34-981-522089; e-mail: diego@fmmeteo.usc.es; http://chaos.usc.es 
Here, we focus on a discrete reaction-diffusion model such that its local dynamics (reaction term) is bistable, with two stable fixed points. Particularly important examples of bistable dynamics are found in optics [5,6], chemical systems [7], and biology [3,8,9]. The main interest of these systems lies in the behaviour of the fronts that constitute the boundaries of the domains of both (stable) states. Intuitively, the front will move from the most to the less stable state, enlarging a domain and shrinking the other. In fact, great attention has been devoted to the phenomenon of 'propagation failure' or 'pinning' [10-15], because it is usual that in discrete systems some threshold must be surpassed to achieve propagation.

But, although one could expect that propagation does not succeed in the case of symmetric bistability, it was demonstrated that such possibility exists. Hagberg and Meron [16] studied a continuous bistable reaction-diffusion system, the FitzHugh-Nagumo model, showing that, in the symmetric case, propagation occurred after a symmetry breaking front bifurcation. In this scenario, the front breaks its symmetry through a pitchfork bifurcation, at the same time that propagation is initiated. Also, an analogous bifurcation was found for the complex GinzburgLandau equation [17] with the name of nonequilibrium Ising-Bloch bifurcation.

In a recent work, we have reported [18] the existence of front propagation in a discrete symmetric bistable medium. The transition is exclusive for a discrete system and it is possible thanks to the multi-variable nature of the local dynamics. One variable systems are not able to break symmetry [10] even if the function that describes the local dynamics is not continuous [19]. It was shown that the mechanism leading to propagation is not a pitchfork bifurcation. Contrastingly, it consists on a Hopf bifurcation followed by a global bifurcation, that is equivalent to a gluing bifurcation of cycles. In correspondence, the velocity of the front shows a logarithmic dependence close to the onset. The aim of the present contribution is two-fold. On one hand, a more detailed analysis of the transition is undertaken, explaining which is the role of the stationary states of the system. On the other hand, the new features of the transition when a saddle-focus fixed point mediates the gluing bifurcation are investigated.

The paper is organized as follows: Section 2 presents the model. In Sec. 3 an overview of the transition is presented. In Sec. 4 a reduced cylindrical coordinate system is defined, and the mechanism of gluing of cycles underlying the transition between oscillating and travelling fronts is explained. In Sec. 5, several non-trivial front dynamics are shown, including the chaotic motion of the front. Finally, Sec. 6 is devoted to further considerations, while in Sec. 7 we present the conclusions.

\section{The system}

We consider a discrete reaction-diffusion equation in one dimension:

$$
\dot{\mathbf{r}}_{j}=f\left(\mathbf{r}_{j}\right)+\frac{D}{2} \Gamma\left(\mathbf{r}_{j+1}+\mathbf{r}_{j-1}-2 \mathbf{r}_{j}\right)
$$


where $D$ accounts for the coupling strength between neighbours, and the coupling matrix $\Gamma$ says which variables get coupled. In what concerns the local bistable dynamics, we deal with the wellknown Lorenz oscillator [20] as unit cell. It exhibits several characteristic behaviours depending on its internal parameters [21]: monostability, bistability, limit cycle, 'butterfly chaos', 'noisy periodicity', etc. We consider the range where the system contains two stable symmetry related fixed points (bistability). The coupling matrix $\Gamma$ is taken with all elements zero except one: $\Gamma=\gamma_{k l}=\delta_{k 2} \delta_{l 2}$. Therefore, the equations describing the temporal evolution of the array are:

$$
\begin{aligned}
& \dot{x}_{j}=\sigma\left(y_{j}-x_{j}\right) \\
& \dot{y}_{j}=r x_{j}-x_{j} z_{j}-y_{j}+\frac{D}{2}\left(y_{j+1}+y_{j-1}-2 y_{j}\right) \quad j=1, \ldots, N \\
& \dot{z}_{j}=x_{j} y_{j}-b z_{j}
\end{aligned}
$$

The standard values are chosen for the $\sigma$ and $b$ parameters: $\sigma=10$ and $b=8 / 3$. Then, the

Lorenz system contains two stable foci $C_{ \pm}=( \pm \sqrt{b(r-1)}, \pm \sqrt{b(r-1)}, r-1)$ for $r$ in the range (1.35, $\left.r_{H}\right)$; at $r=r_{H} \approx 24.74, C_{ \pm}$become unstable through a subcritical Hopf bifurcation. Also, it is important to note that there exists a saddle fixed point located at the origin for $r>1$.

A fourth-order Runge-Kutta method, with time step 0.01, was used to integrate Eq. (2). A steplike initial condition is imposed to the system. Results on front propagation do not depend on the boundary conditions provided that the array is large enough. Thus, if one wishes the system evolve for long time in the regime of travelling front, one should move the boundary whilst the front propagates. Also, one may imposed to the system periodic boundary conditions, with two fronts diametrically opposed as initial conditions. If the initial condition satisfies $x_{j}=-x_{j+N / 2}$, $y_{j}=-y_{j+N / 2}, z_{j}=z_{j+N / 2}$, both fronts propagate always in the same direction, avoiding its mutual annihilation.

\section{Standing, oscillating and travelling fronts}

Three main front dynamics are found when varying the parameter $r\left(r<r_{H}\right)$, and the coupling strength $D(D>0)$. We distinguish among standing (static), oscillating and travelling fronts. In Fig. 1, a centered step-like initial condition is imposed to the system with free ends and $r=14$; two different non-static regimes are achieved depending on the value of the coupling strength $D$. Note that the Lorenz model is symmetric and the coupling that appears in Eq. (2) destroys this local symmetry but preserves the global reflection (or $\mathbf{Z}_{2}$ ) symmetry:

$$
\left(x_{1}, y_{1}, z_{1}, \ldots, x_{N}, y_{N}, z_{N}\right) \longrightarrow\left(-x_{1},-y_{1}, z_{1}, \ldots,-x_{N},-y_{N}, z_{N}\right)
$$

Therefore, for $N$ large enough, both senses of propagation are equally likely. A diagram of the different regions on the $r-D$ plane is shown in Fig. 2. It may be seen that static fronts are found for $D$ and/or $r$ small, whereas travelling fronts appear for large $D$ and $r$. Also, it is 

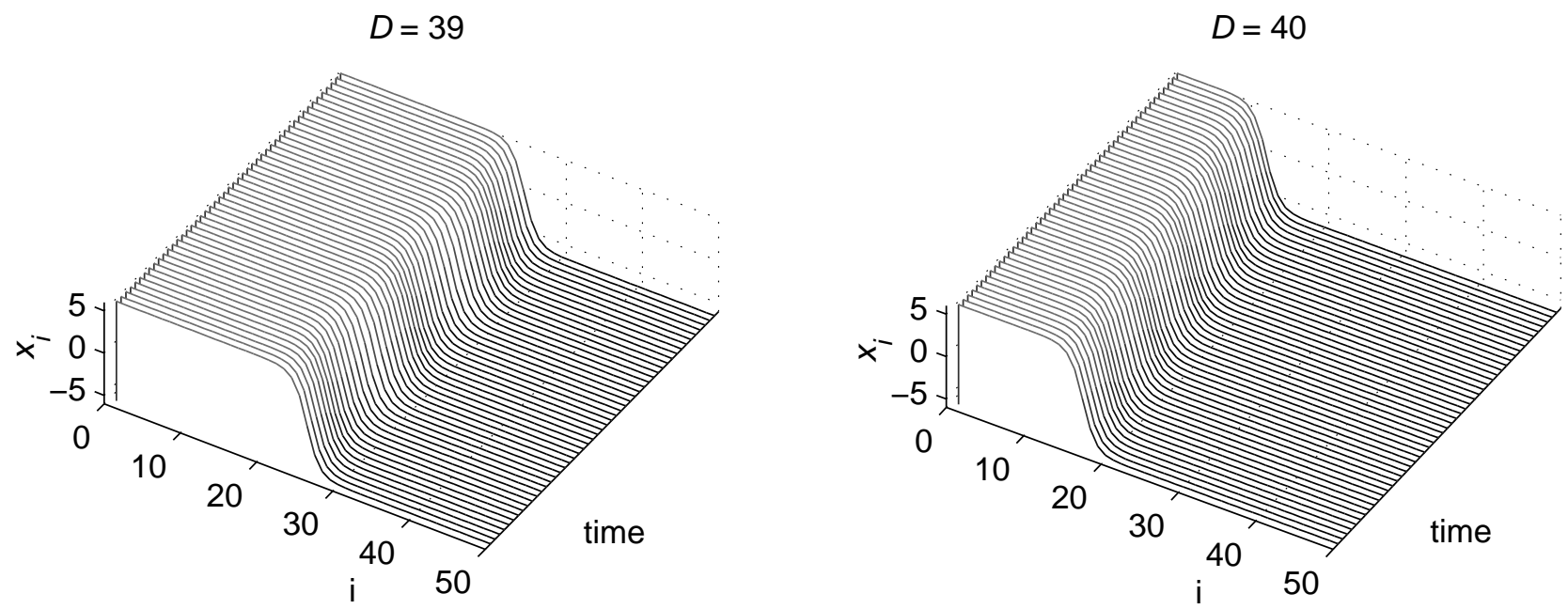

Fig. 1. Spatio-temporal evolution of the front for two different values of the coupling strength $D$ and $r=14$. A step-like initial condition is imposed for an open array of $N=50$ oscillators. When the coupling surpasses the critical value $D=D_{t h} \approx 39.63$ the front propagates through the array shifting the oscillators from $C_{+}$to $C_{-}$. The time interval shown is 100 time units.

significative that oscillating fronts always exist between the standing and the travelling fronts regions. The line $\left(D_{o s}\right)$, that defines the boundary between the standing and the oscillating regions, approach $D=0$ as $r \rightarrow r_{H}$, since the nature of the fixed points $\left(C_{ \pm}\right)$deeply influences the dynamics of the front. This is not very surprising, but indicates that oscillating fronts will be usually found in bistable systems with stable foci, rather than nodes. Somehow, the stability properties of the fixed points are transmitted to the front. Also, it is significative that $D_{t h}$ (the threshold for travelling fronts) seems to diverge asymptotically at $r=r_{\infty} \approx 13.5$.

Instead of visualizing the system as in Fig. 1, we show now which is the behaviour of the different units when the coupling is increased from zero. It is clear that for $D=0$ the step-like solution that we were considering consists of $\mathbf{r}_{j}=C_{+}, j=1, \ldots, 25$ and $\mathbf{r}_{j}=C_{-}, j=26, \ldots, 50$ $(N=50)$. As the coupling increases this solution can be smoothly continued. But there exist two constrains for the stationary solutions, that come from Eq. (2):

$$
x_{j}=y_{j}, \quad z_{j}=\frac{x_{j} y_{j}}{b} \quad \forall j \in\{1,2, \ldots, N\}
$$

Therefore, all the units lie on a parabola that passes through $C_{ \pm}$and zero. However, when $D_{o s}$ is reached the front undergoes a Hopf bifurcation, and all the units start to oscillate leaving the mentioned parabola. The projection of the parabola onto the plane $(x, y)$ is a straight line, the bisectrix of the first and third quadrants. However, to better visualize oscillations out of the parabola, in Fig. 3 we performed a $45^{\circ}$ rotation of the reference system. Obviously those units closer to the front oscillate with larger amplitude that those located far from the front that are almost insensitive to the bifurcation. When $D_{t h}$ is reached a multiple collision occurs; the orbits of neighbouring units collide creating two "channels" going from $C_{ \pm}$to $C_{\mp}$. Like in the symmetric FitzHugh-Nagumo model studied by Hagberg and Meron [16], the travelling front is 


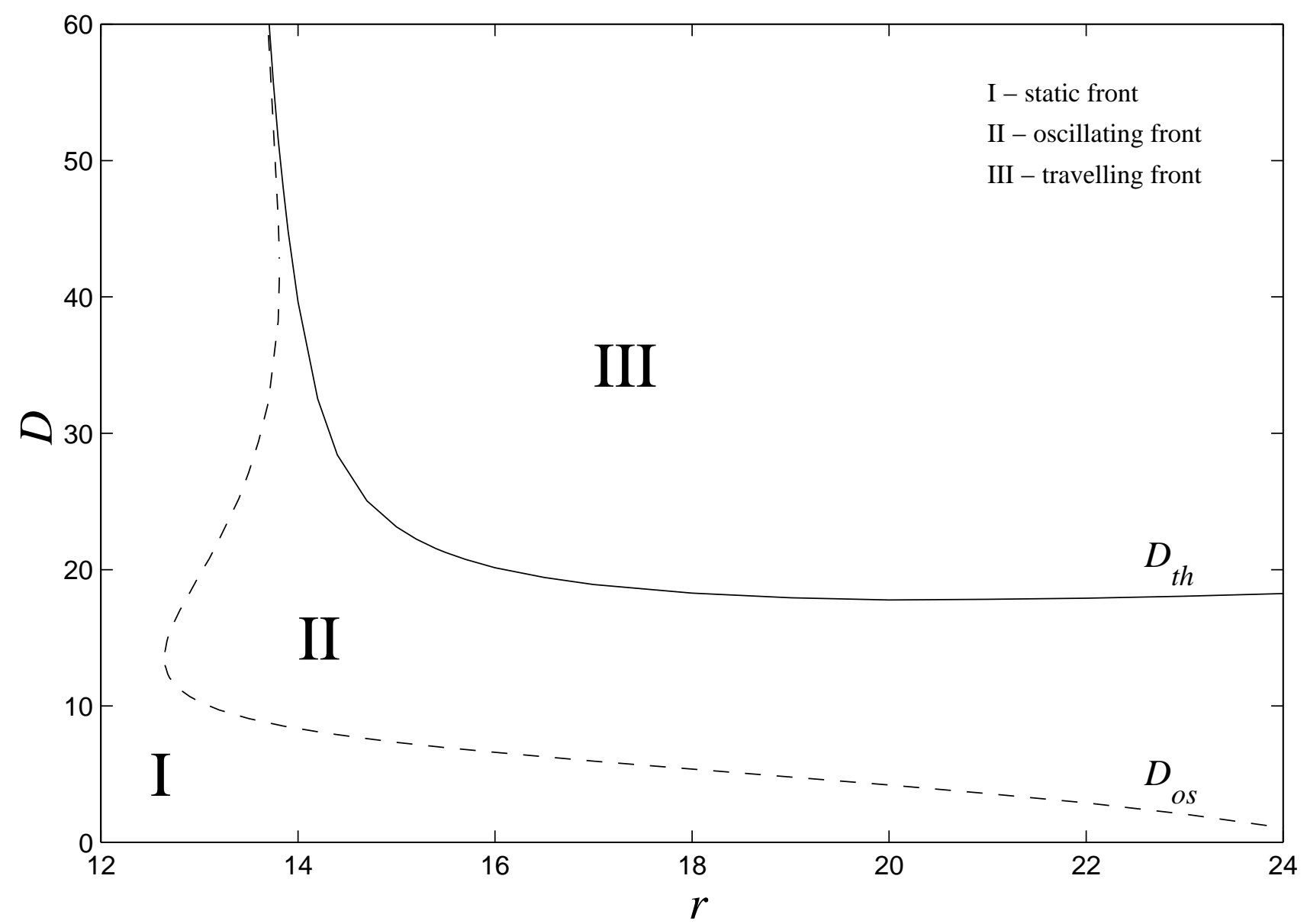

Fig. 2. Three main types of dynamics are distinguished as a function of $r$ and $D$. The boundaries among them are the critical lines $D_{o s}$ and $D_{t h}$.

not symmetric. However, in our case the mechanism leading to propagation is not a pitchfork bifurcation, instead, a Hopf bifurcation that creates the oscillating front is the precursor of the travelling front.

The description provided by Fig. 3 is illustrative, but it is incomplete if one does not realize that besides the static solution that loses its stability through a Hopf bifurcation, there must exist another (unstable) stationary state that mediates the multiple collision of cycles. Therefore, one must search the stationary solutions monotonic in $\left\{x_{j}\right\}$ and $\left\{y_{j}\right\}$. It is not difficult to find out that only two monotonic solutions, called stable and unstable dislocations, exist (discarding spatial translations). They are the continuation of the solutions in the uncoupled limit: $\left(\ldots, C_{+}, C_{+}, C_{-}, C_{-}, \ldots\right)$ and $\left(\ldots, C_{+}, C_{+}, \mathbf{0}, C_{-}, C_{-}, \ldots\right)$. We shall refer to them as $A$ and $B$-state, respectively, and Fig. 4 shows a sketch of them. It is the $B$-state which mediates the transition at $D_{t h}$, and it will be shown below that the stability properties of this solution will determine important features of the onset of the wavefronts. 

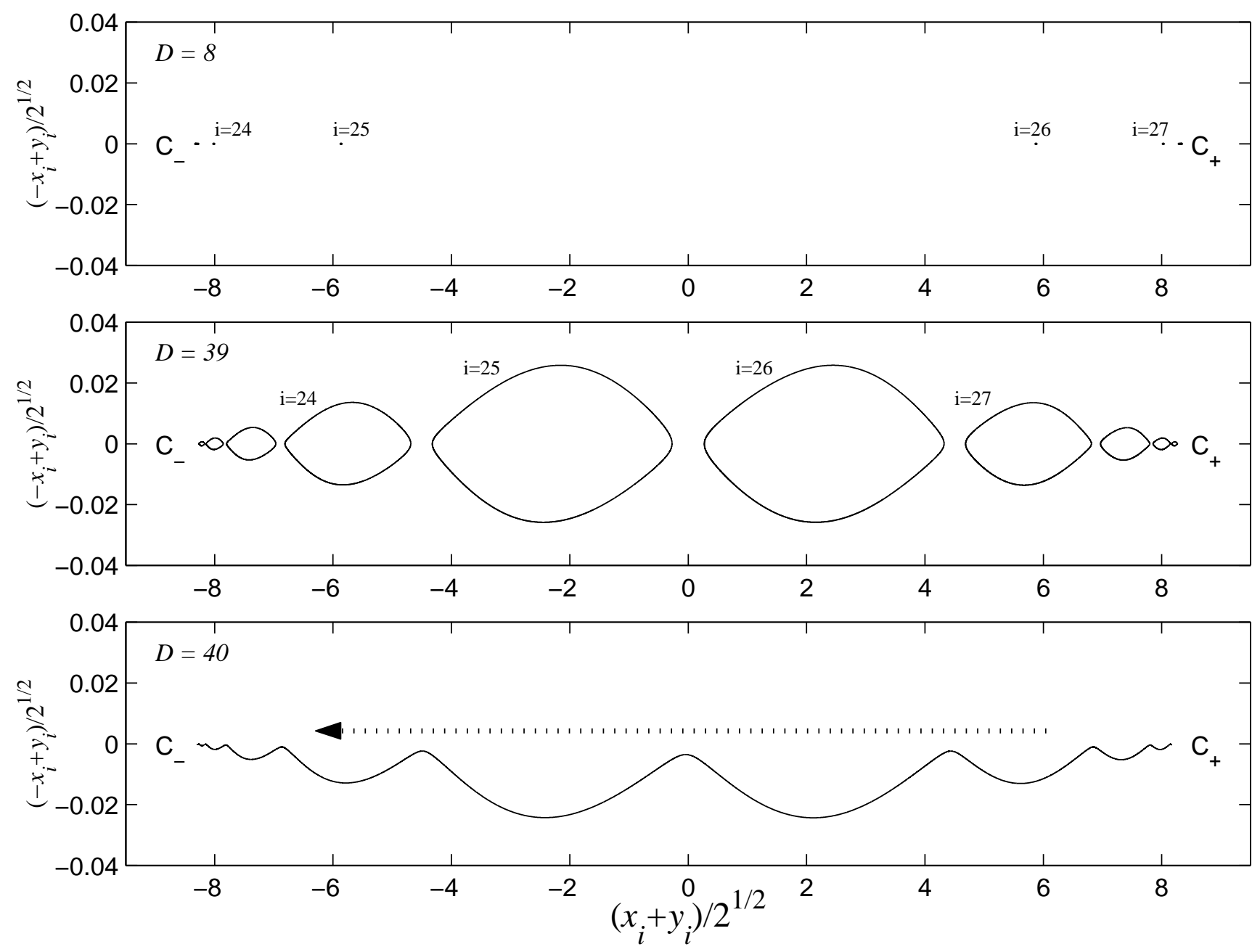

Fig. 3. Projections onto the reference system $\left(\left(x_{i}+y_{i}\right) / \sqrt{2},\left(-x_{i}+y_{i}\right) / \sqrt{2}\right)$ of all the oscillators of the array with step-like configuration and $r=14$. From top to bottom, standing, oscillating, and travelling cases are shown. In the travelling case, the line is the trajectory followed by each oscillator going from $C_{+}$to $C_{-}$as the front advances.

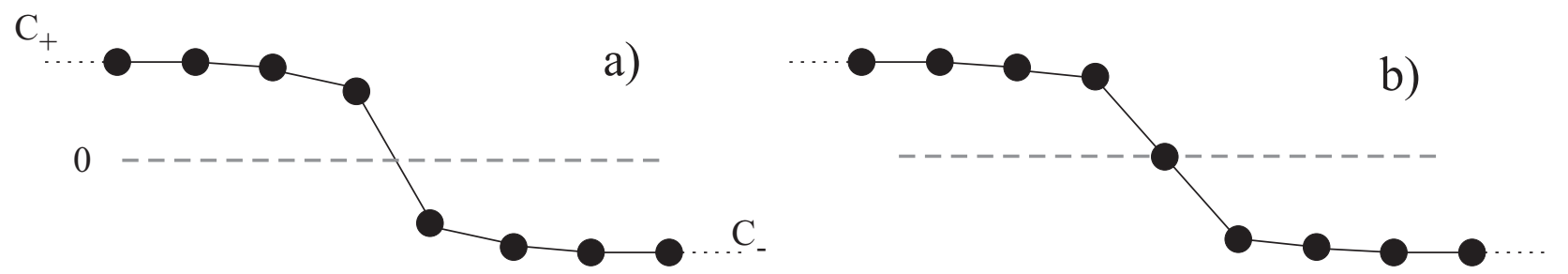

Fig. 4. A sketch of the two monotonically decreasing stationary solutions for $D>0$. a) $A$-state, continuation of the state $\left(\ldots, C_{+}, C_{+}, C_{-}, C_{-}, \ldots\right)$ at $D=0$. b) $B$-state, continuation of the state $\left(\ldots, C_{+}, C_{+}, \mathbf{0}, C_{-}, C_{-}, \ldots\right)$ at $D=0$. 


\section{Gluing of cycles}

In this section we demonstrate how the transitions presented in the previous section may be described in the context of a gluing bifurcation, in which two limit cycles become a two-lobed cycle by involving an intermediate saddle point. The gluing bifurcations is usual in systems with $\mathbf{Z}_{2}$ symmetry $[22,23]$, because provided the existence of the symmetry it becomes a codimensionone bifurcation.

\subsection{Cylindrical coordinates}

Figure 3 shows some kind of collective motion when the front oscillates. Then, the whole set of variables $\left(x_{j}, y_{j}, z_{j}\right)$ is discarded, and instead, we chose to build a reduced phase space to study the dynamics. Notice that if the medium is infinite, there exists a perfect symmetry under translation along the array. With this in mind, we define two auxiliary variables $\xi$ and $\eta$, that have sense for large enough $N$ and when the front is far enough from the boundaries:

$$
\begin{aligned}
& \xi=\frac{1}{\sqrt{2}} \sum_{j=1}^{N} x_{j}+y_{j} \quad \bmod (2 \sqrt{2 b(r-1)}) \\
& \eta=\frac{1}{\sqrt{2}} \sum_{j=1}^{N}-x_{j}+y_{j} .
\end{aligned}
$$

Variable $\xi$ accounts for the propagation of the front and is not bounded by default, then it

must be defined within the range $\Delta \xi=[2 \sqrt{b(r-1)}+2 \sqrt{b(r-1)}] / \sqrt{2}=2 \sqrt{2 b(r-1)}$, that is the magnitude that $\xi$ increases or decreases when the front moves one position along the array. On the other hand, $\eta$ is defined so that it is bounded under front propagation, notice that it is defined from Eq. (4). If the medium consists of a number $N$ even of units, the static solutions are located in this cyclic phase space in:

$$
\begin{aligned}
& A: \xi=\eta=0 \\
& B: \xi= \pm \sqrt{2 b(r-1)}, \eta=0
\end{aligned}
$$

If $N$ is odd both solutions exchange their coordinates. In what follows, we only take $N$ even, unless it is specified.

The transition shown in Fig. 3 is now shown in the cylindrical phase space in Fig. 5. Notice that when the front is static, we have an equilibrium point at $\xi=\eta=0$ ( $A$-state). When the front oscillates a limit cycle exists, and finally when the coupling goes beyond $D_{t h}$ we find two symmetry related limit cycles, that turn around the cylindrical phase space in opposite 

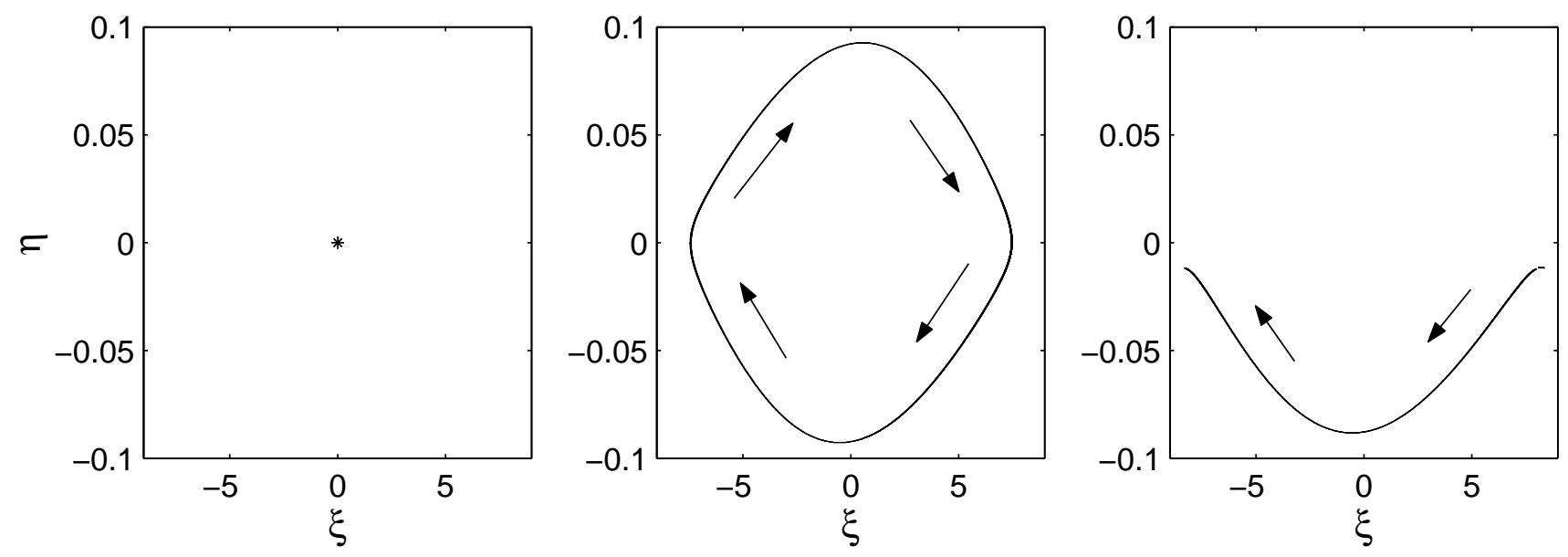

Fig. 5. Evolution on the reduced cylindrical phase space spanned by $\xi$ and $\eta$ for three different values of $D$ and $r=14$; from left to right: standing, oscillating, and travelling front. At $D=D_{t h} \approx 39.63$ a double homoclinic connection to the $B$-state arises.

directions. The situation is quite similar to a pendulum where libration corresponds now to oscillation, and rotation of the pendulum corresponds to propagation. Thus, at $D=D_{t h}$ there exist two homoclinic loops that connect the $B$-state with itself (called separatrices for the pendulum). This bifurcation is called gluing bifurcation because it involves the collision of two cycles to create another; although in our case it could be more appropriate to talk of a inverse gluing or a splitting bifurcation.

Nonetheless, it is better to visualize our gluing bifurcation in $\mathbb{R}^{2}$. A possible transformation consists, topologically, in what follows: First of all, cut the cylinder with two planes perpendicular to its axis in such a way that the saddle point and the heteroclinic orbits stand between both planes. Then compress both circumferences that limit the "cylinder" inbetween to a point. Thus, we have got an object topologically equal to a sphere. These two steps could be substituted by making the section of the cylinder equal to zero at $\eta= \pm \infty$ and then making a transformation that makes our object finite. The last step is to make a hole at $\xi=\eta=0$ and deform what remains into a plane.

In Fig. 6 the sketch of a gluing bifurcation equivalent to the one shown in the cylindrical phase space (Fig. 5) is depicted. Notice that for $D<D_{t h}$ there exist an orbit that approaches twice per period close to the saddle point (the $B$-solution), whereas for $D>D_{t h}$ two symmetry related cycles, corresponding to both senses of propagation, coexist.

\subsection{Velocity of the front as a function of $D-D_{\text {th }}$}

The collision (and subsequent destruction) of a periodic orbit with a saddle, called saddle-loop bifurcation, is characterized by a logarithmic lengthening of the period of the cycle [24]. For the scenario depicted in Fig. 6, we expect to find the following dependencies for the periods 

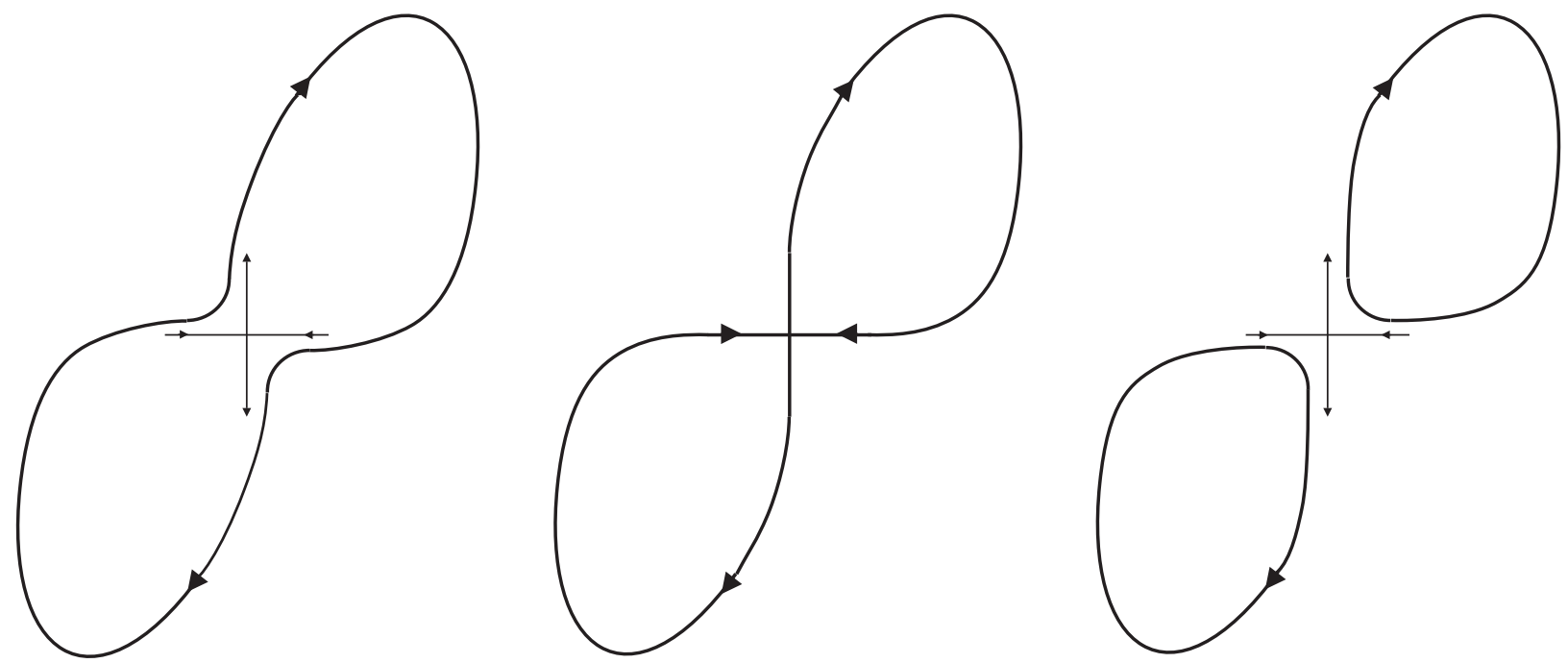

Fig. 6. Schematic of a (inverse) gluing bifurcation on $\mathbb{R}^{2}$. For $D<D_{t h}$ the trajectory approaches twice per cycle the saddle point and the front oscillates. Beyond the critical value $D=D_{t h}$ two symmetry related orbits, corresponding to both senses of propagation of the front, appear. $\lambda_{u}$ and $\lambda_{s}$ stand for the eigenvalues of the saddle fixed point at homoclinicity.

$\left(T_{1,2}\right)$ in a neighbourhood of $D_{t h}$ :

$$
\begin{gathered}
T_{1}=a_{1}-\frac{2}{\lambda_{u}} \ln \left(D_{t h}-D\right) \\
\frac{1}{c}=T_{2}=a_{2}-\frac{1}{\lambda_{u}} \ln \left(D-D_{t h}\right),
\end{gathered}
$$

where $\lambda_{u}$ is the unstable eigenvalue of the saddle fixed point. Taking into account that one turn around the cylinder is equivalent to the movement of the front in one cell, it is clear that $T_{2}$ is the inverse of the velocity of the front $(c)$. In the equation for the period $T_{1}$ a factor 2 appears because the orbit approaches twice per cycle to the neighbourhood of the saddle point. Moreover, it is expected that the 'fast dynamics' (the motion far from the saddle) contained in variables $a_{1,2}$ will be approximately the same at both sides of the transition, and then, $a_{1} \approx 2 a_{2}$. In Ref. [18] it was shown that at $r=14$, the behaviour of $T_{1} / 2$ and $c^{-1}$ agrees with the predictions given by Eqs. $(9,10)$, and we show it here for $r=16$ in Fig. 7 .

Notice that the velocity of the front grows quite abruptly from zero at $D=D_{t h}$. The derivative of $c=c(D)$, near $D_{t h}$ is, according to Eq. (10):

$$
\frac{d c}{d D}=\frac{1}{\lambda_{u}\left(D-D_{t h}\right)}\left(\frac{1}{a_{2}-\frac{1}{\lambda_{u}} \ln \left(D-D_{t h}\right)}\right)^{2} \quad \stackrel{D \rightarrow D_{t h}}{\longrightarrow} \infty .
$$



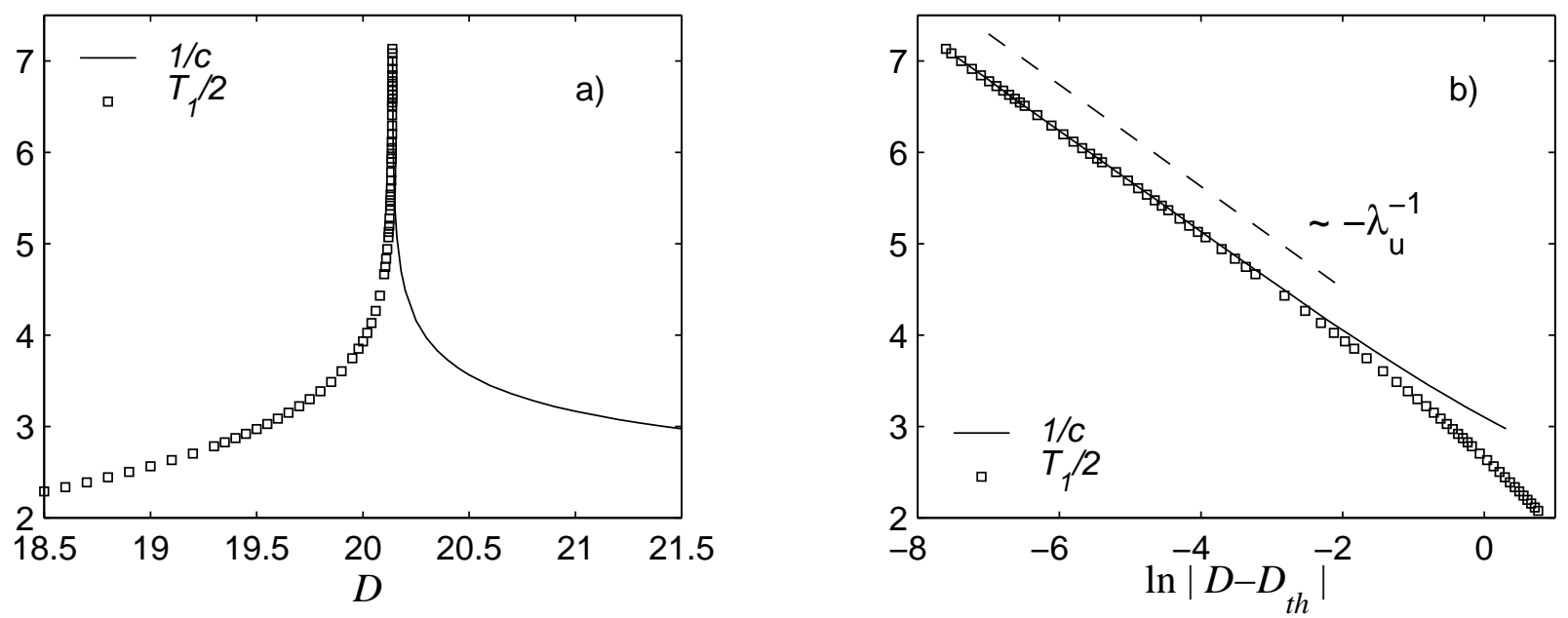

Fig. 7. $1 / c$ (solid line) and $T_{1} / 2$ (squares) as a function of $D-D_{t h}$ (a) and $\ln \left(\left|D-D_{t h}\right|\right.$ ) (b) for $r=16$. The behaviour agrees with that predicted by Eqs. (9) and (10). The critical coupling is found to be $D_{t h}^{r=16}=20.13952$. In (b) a straight line with slope $-\lambda_{u}^{-1}$ is depicted, being $\lambda_{u}=1.7959$ the unstable eigenvalue of the $B$-state computed numerically for an array of $N=21$ units.

\subsection{Quantitative analysis}

In order to verify, not only qualitatively, but quantitatively the tendency of $T_{1}$ and $c$ when $D \rightarrow D_{t h}$ we computed numerically the value of the eigenvalues of the $B$-state at $D=D_{t h}$ for finite $N$. To preserve the symmetry, when computing the eigenvalues, the number of units $N$ was chosen to be odd, with the central unit located at the origin. The jacobian matrix $(J)$ for a discrete reaction-diffusion model (see Eq. (1)) exhibits the following tridiagonal structure:

$$
J=\left(\begin{array}{cccccc}
\mathbf{H}_{1} & \mathbf{D} & \mathbf{0} & \cdots & \mathbf{0} & \mathbf{0} \\
\mathbf{D} & \mathbf{H}_{2} & \mathbf{D} & \cdots & \mathbf{0} & \mathbf{0} \\
\mathbf{0} & \mathbf{D} & \mathbf{H}_{3} & \cdots & \mathbf{0} & \mathbf{0} \\
\vdots & \vdots & \vdots & \ddots & \vdots & \vdots \\
\mathbf{0} & \mathbf{0} & \mathbf{0} & \cdots & \mathbf{H}_{N-1} & \mathbf{D} \\
\mathbf{0} & \mathbf{0} & \mathbf{0} & \cdots & \mathbf{D} & \mathbf{H}_{N}
\end{array}\right),
$$

which, according to Eq. (2) has got the components:

$$
\mathbf{H}_{j}=\left(\begin{array}{ccc}
-\sigma & \sigma & 0 \\
r-z_{j} & -1+D & -x_{j} \\
y_{j} & x_{j} & -b
\end{array}\right), \quad \mathbf{D}=\left(\begin{array}{ccc}
0 & 0 & 0 \\
0 & -D / 2 & 0 \\
0 & 0 & 0
\end{array}\right),
$$



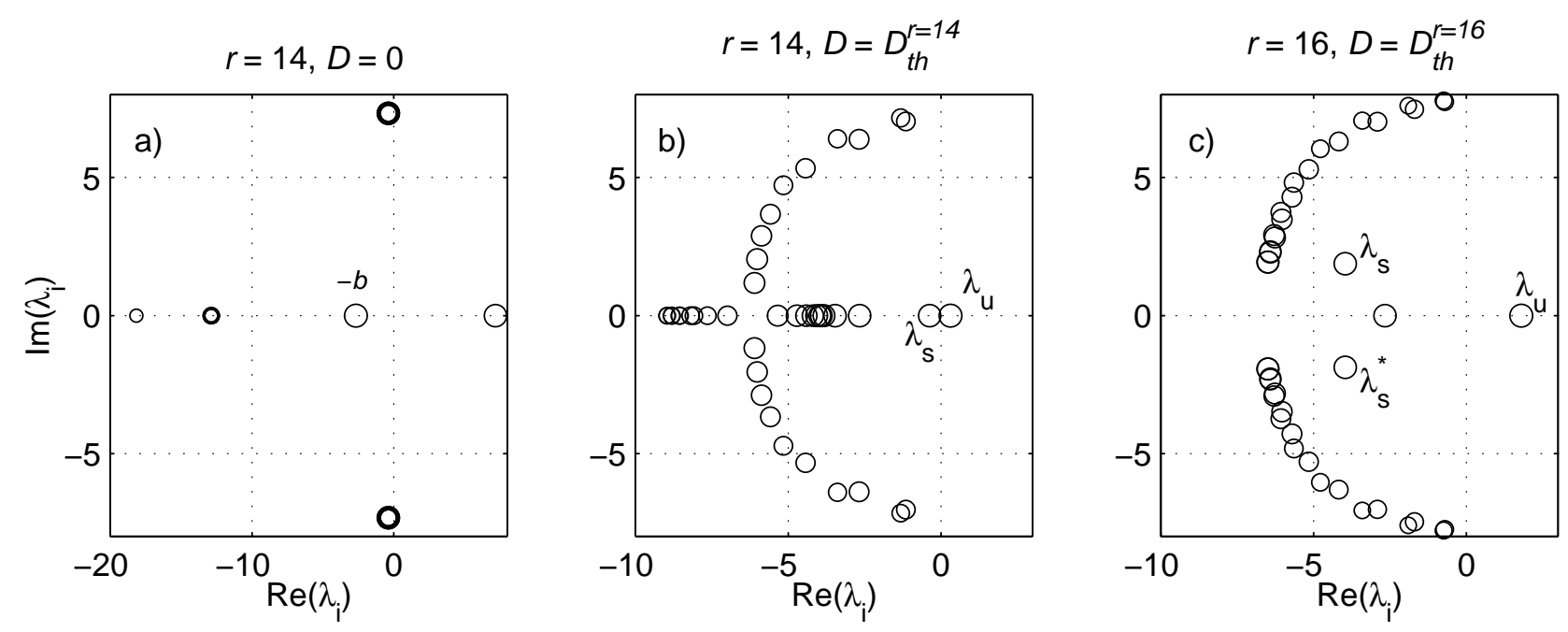

Fig. 8. Eigenvalues of the $B$-state for different values of $D$ and $r$.

recall that $x_{(N+1) / 2}=y_{(N+1) / 2}=0$. Therefore, $-b$ is always an eigenvalue with trivial eigenvector $(0,0, \ldots, 0,1,0, \ldots, 0)^{T}$. Using the program MATLAB we obtained the eigenvalues for an array of $N=21$ units, that we expect to be large enough to inform us about the properties of a front in an infinite medium, as long as the front is a very localized structure.

Figure 8 shows the results for three values of the parameters $r$ and $D$. Fig. 8 (a) shows the eigenvalues $\left(\lambda_{i}\right)$ for $D=0$ and $r=14$, which illustrates the meaning of the different eigenvalues. There are three simple eigenvalues corresponding to the oscillator located in the center of the array whose coordinates are $x_{(N+1) / 2}=y_{(N+1) / 2}=z_{(N+1) / 2}=0$, recall that we are considering a $B$-state. Moreover, there are three $(N-1)$-degenerated eigenvalues, corresponding to the oscillators with coordinates $C_{ \pm}$. Eigenvalues are depicted with circles of different sizes to better observe degeneracy. When $D$ grows above zero degeneracy is lost. In Fig. 8 (b), we graph the eigenvalues for $D=D_{t h} \approx 39.63$ (only those eigenvalues with $\operatorname{Re}\left(\lambda_{i}\right)>-10$ are shown). The unstable eigenvalue corresponds to the fixed point at the origin of a Lorenz oscillator. There is also a slightly negative eigenvalue $\left(\lambda_{s}\right)$ that is the leading eigenvalue among the whole spectrum of negative eigenvalues.

A different scenario is found for $r=16$, as shown in Fig. 8 (c). In this case, it is not so clear which stable eigenvalue must be considered as the leading one in the gluing process. There are several eigenvalues making up two lines at both sides of the real axis in the complex plain, but these are not relevant, as long as they are the eigenvalues associated with the stability properties of each domain at both sides of the front where quasi-homogeneous domains around $C_{ \pm}$exist. As well, if the eigenvalue located at $-b$ is considered, its eigenvector becomes trivial and it cannot be expected to participate in the gluing bifurcation. Then, the solitary eigenvalue denoted by $\lambda_{s}(=\rho+i \omega)$ is the one (with its complex conjugate $\lambda_{s}^{\star}$ ) that defines the leading (twodimensional) stable manifold. In fact, by increasing continuously $r$ along $D_{t h}$, the eigenvalue $\lambda_{s}$ could be followed directly from that obtained for $r=14$ (see Figs. $8(\mathrm{~b}, \mathrm{c})$ ). According to the statements exposed above, the graph of $\eta$ vs. $\xi$, for $D$ close to $D_{t h}$ and $r=16$, shows some 


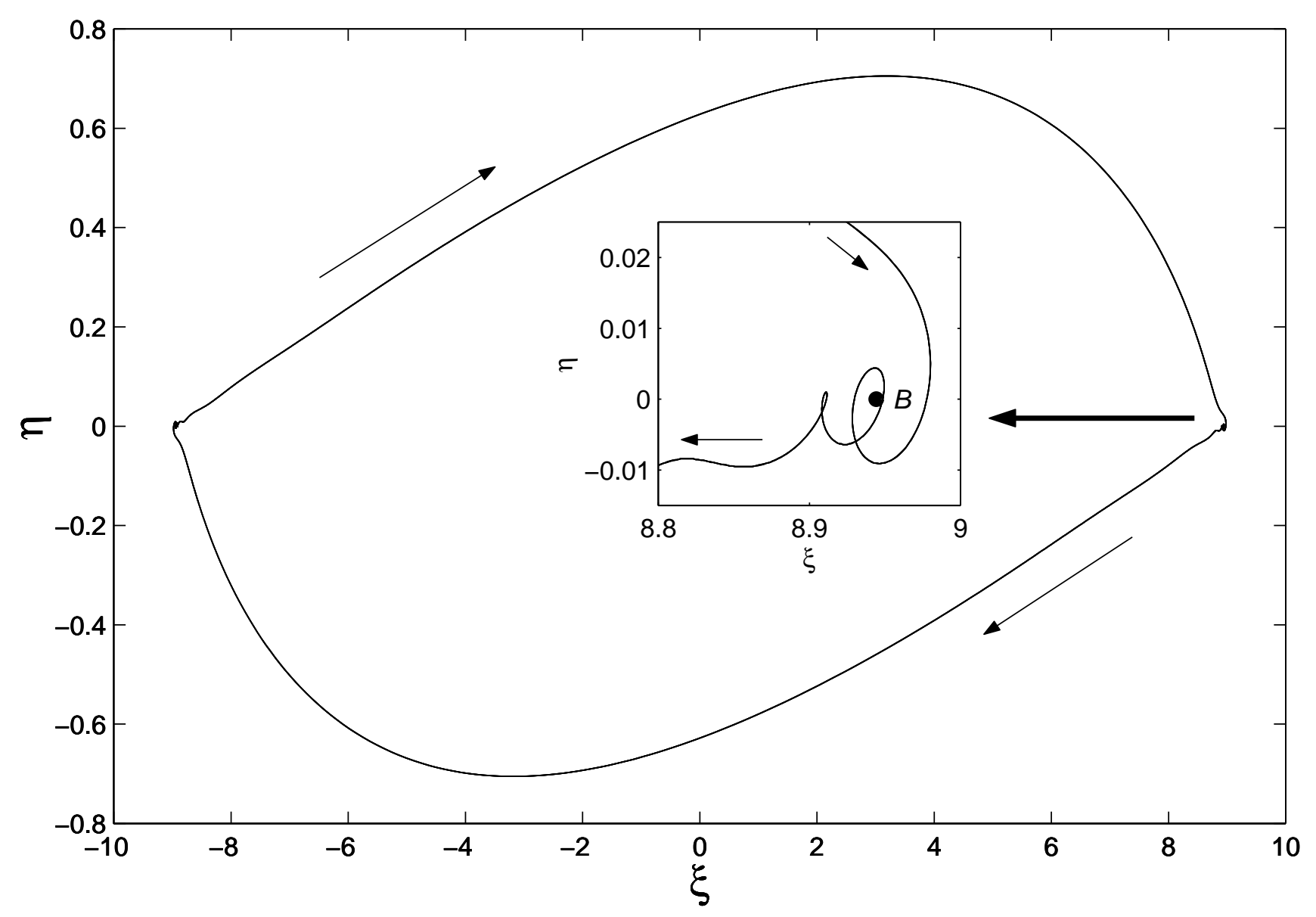

Fig. 9. Projection onto $(\xi, \eta)$ at $r=16, D=20.136<D_{t h} \approx 20.1395$. In the inset, the spiraling approach of the trajectory to the $B$-state (filled circle) may be observed.

spiraling ${ }^{2}$ when the trajectory approaches the $B$-state. Figure 9 shows this effect that confirms that now the gluing is mediated by a saddle-focus. The cyclic definition of $\xi$ has been relaxed in order to get a better observation of the spiral trajectory near the $B$-state. Nonetheless, if one looks at the asymptotic properties of the oscillation period of the front and its velocity, no difference with the case of the planar connection is found. So, Fig. 7 already confirmed the Eqs. (9) and (10), and the slopes agree with the numerical value: $\lambda_{u} \approx 1.7959$.

A sketch of the gluing bifurcation in the saddle-focus case is shown in Fig. 10. Homoclinic orbits, at $D=D_{t h}$ are denoted by $\Gamma_{0}$ and $\Gamma_{1}$. The process of gluing is drawn in analogy to Fig. 6 . Although, from both figures, one could think that the complex and the real cases are almost equivalent, there exist very fundamental differences between both cases. Thus, the transition from oscillating to travelling fronts may become quite convoluted in the complex case. This is observed for larger values of $r$ and is the subject of the next section.

$\overline{2}$ One should define a new extra variable $\zeta$ to work in a hyper-cylindrical phase space. One could define, for instance, $\zeta=\sum x_{j} y_{j}-b z_{j}$. Nonetheless, we shall continue to work with $\xi$ and $\eta$ only, although keeping in mind that we are projecting the third variable. 

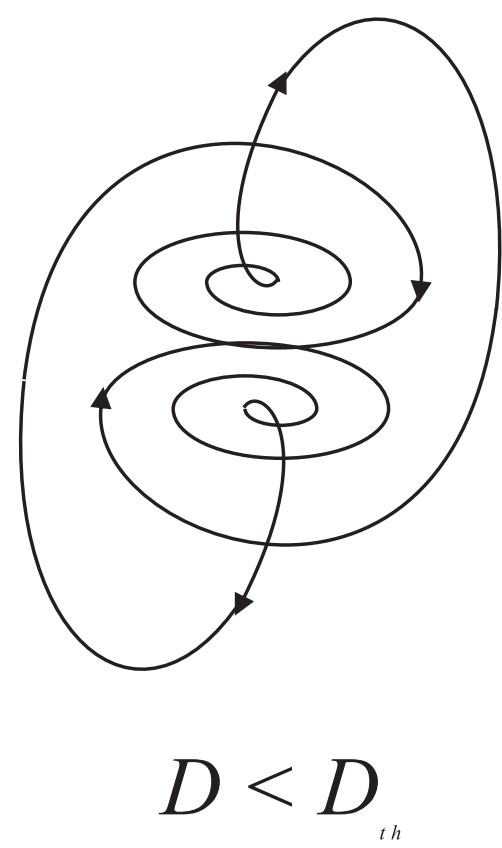

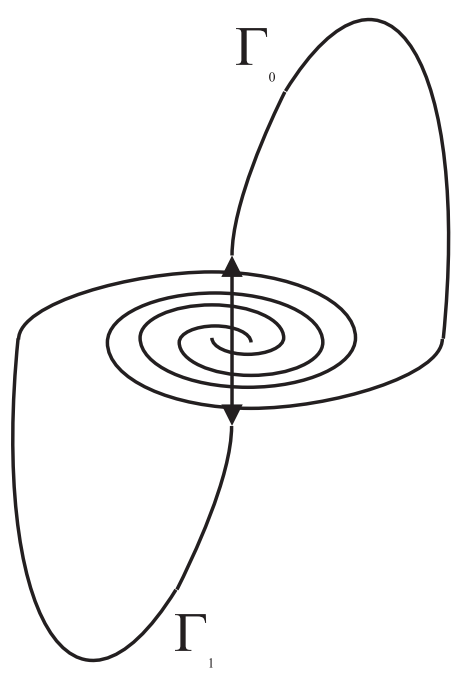

$D=D_{t h}$

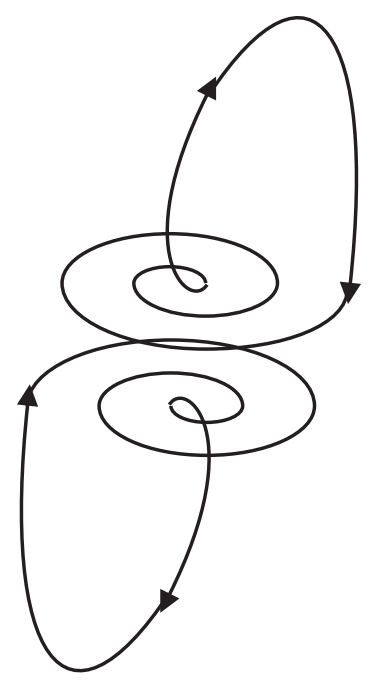

$D>D_{t h}$

Fig. 10. Schematic of a gluing bifurcation mediated by a saddle-focus. Two homoclinic trajectories $\Gamma_{0,1}$ exist at $D=D_{t h}$.

\section{Exotic front dynamics}

In Figure 11 the value of the complex part of the stable leading eigenvalues $(\omega)$, along the line $D=D_{t h}$, is shown as a function of $r$ with a dashed line. It is found that above $r=r_{s f} \approx 15.45$ the stable leading eigenvalues are complex conjugates, and therefore, the gluing bifurcation occurs mediated by a saddle-focus point.

Many works have been devoted to the problem of saddle-loop bifurcations involving a saddlefocus fixed point (see e.g. [24,27,28] and references therein). A deep presentation of this problem is out of the scope of this paper, so we just introduce some of the most relevant results.

For the case of a single homoclinic orbit, occurring at a critical value of the control parameter (e.g. $\mu=0$ ), it was found by Shil'nikov [26] that when the ratio

$$
\delta=-\frac{R e\left(\lambda_{s}\right)}{\lambda_{u}}=-\frac{\rho}{\lambda_{u}}
$$

called saddle index, is less than one, there are a countable infinity of periodic orbits in a neighbourhood of the homoclinic orbit, all of which are saddles. These saddle orbits are created in a sequence of tangent bifurcations at both sides of $\mu=0$, such that the stable branches (provided $\delta>1 / 2$ ) become unstable through period-doubling bifurcations. This scenario, where infinite orbits, with periods ranging from some finite value to an infinite one, arrange in a wiggly curve around the critical value, is known as Shil'nikov wiggle. And it is, with the Lorenz and 


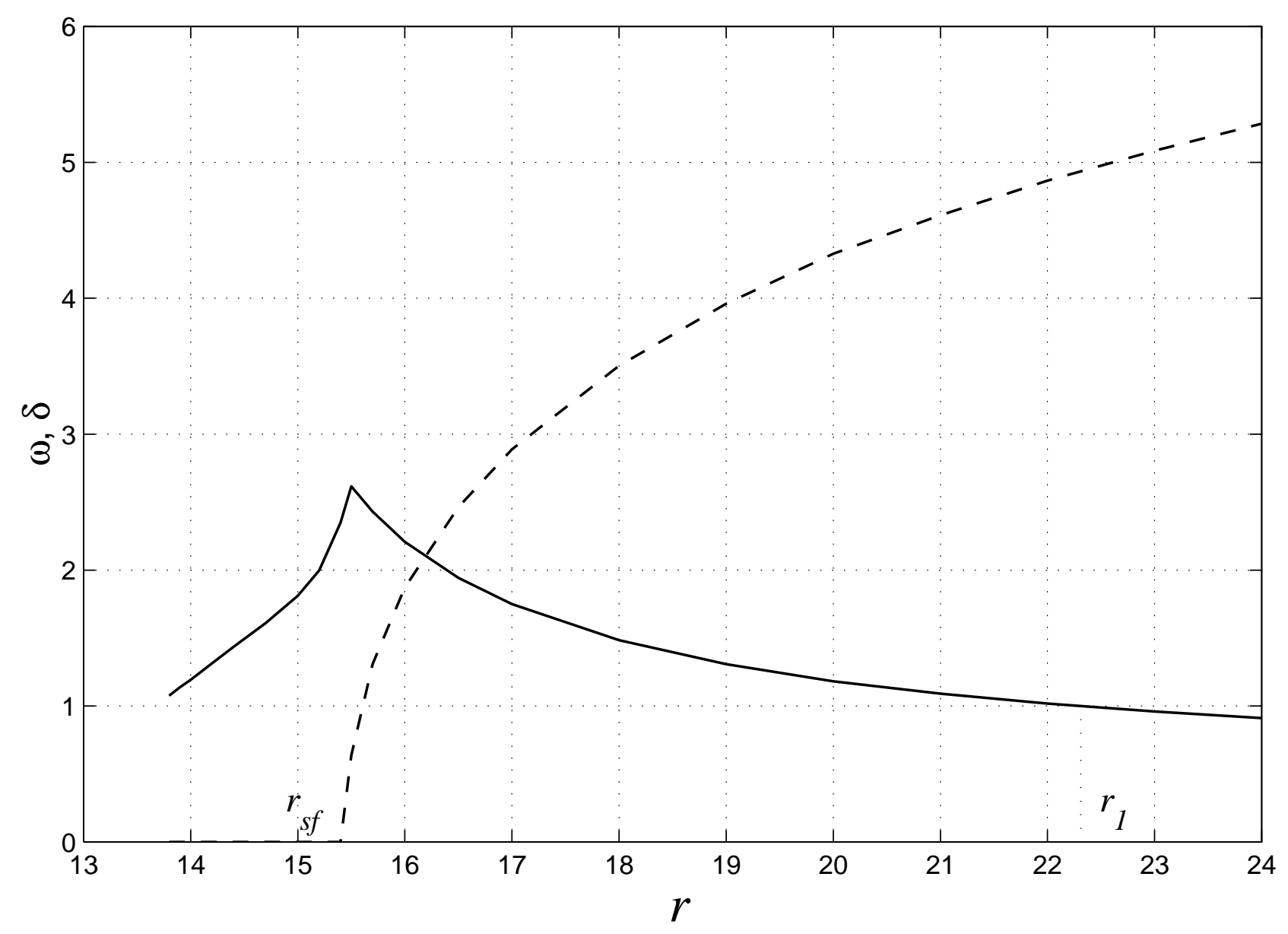

Fig. 11. Complex part of the stable leading eigenvalue $\omega$ (dashed line) of the $B$-state and saddle index $\delta$ (solid line), computed along $D=D_{t h}$ as a function of $r$. For $r>r_{s f}, \omega>0$; and for $r>r_{1}, \delta<1$.

the bifocal mechanisms, one of the universal routes to chaos through homoclinicity $[27,28]$.

However, the results by Shil'nikov apply in a very small neighbourhood of the critical parameter. Therefore, the relationship between the local (theoretical) results and the global (observed) behaviours cannot be stated oversimply. It was shown by Glendinning and Sparrow [29] that the difference between the $\delta<1$ and the $\delta>1$ approaches to homoclinicity may not be easily observed numerically and may not be relevant from a global point of view. In fact, for the lowest branch (the orbit with the lowest period) that already exist far from the critical parameter, deviations from the behaviour predicted by the local analysis are most likely to occur.

One may realize the complexity of the problem if one notices that, besides the Shil'nikov wiggle, there exist subsidiary homoclinic connections. Following the nomenclature of Ref. [29], subsidiary homoclinic orbits are multiple-pulse homoclinic orbits, i.e. orbits that pass several times near the stationary point without achieving homoclinicity.

In case the homoclinic connection is double (two loops), as occurs in the gluing bifurcation, some differences are appreciated with the case described above. The most important point is 

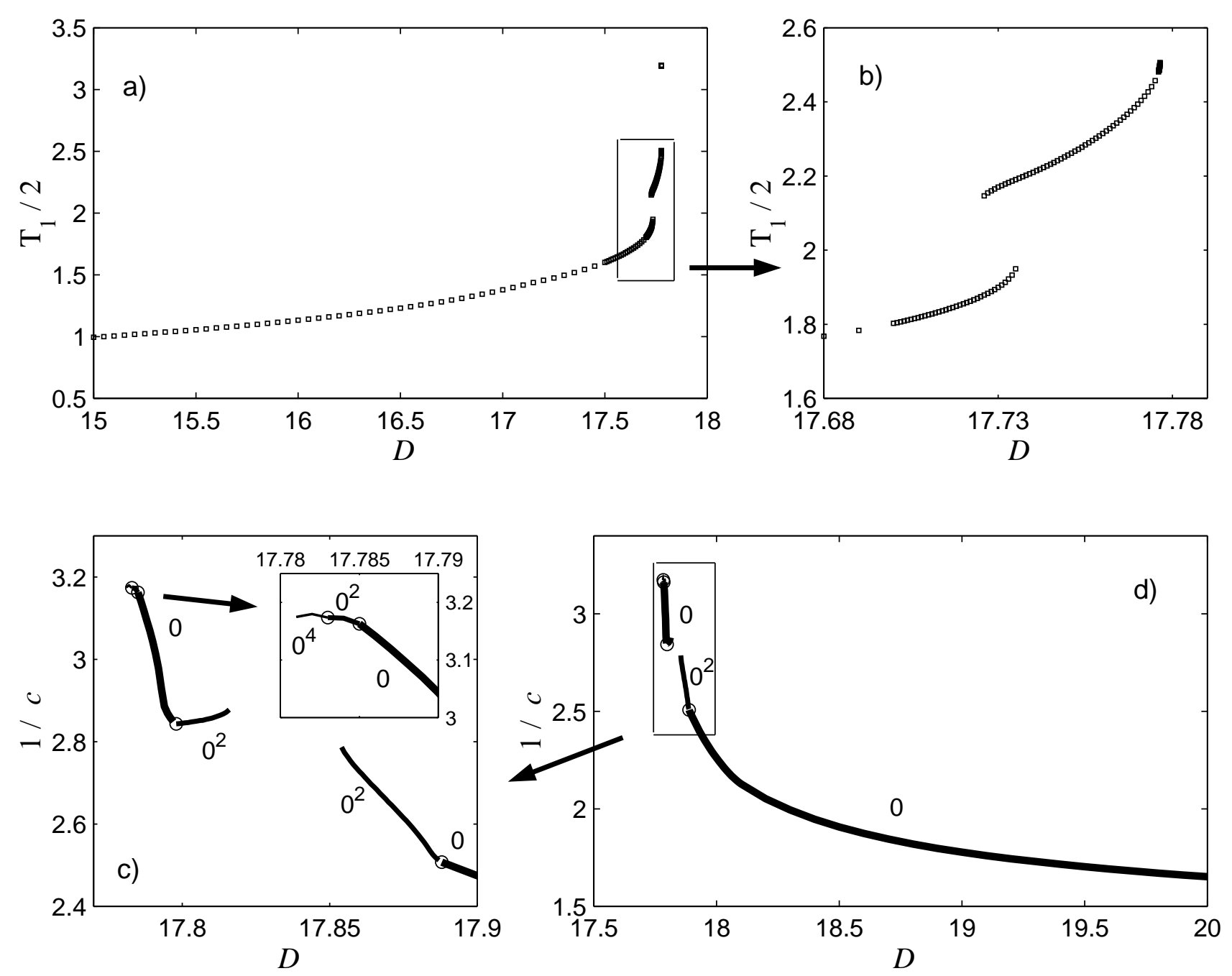

Fig. 12. $1 / c$ (solid line) and $T_{1} / 2$ (squares) as a function of $D$ for $r=20$. Fig. (a) shows that the oscillation period does not grow continuously. An inset, showing one of this jumps can be seen in (b). The velocity of the front -Fig. (d) and inset (c)- is not defined in some regions where chaotic states are found. Period-doublings are marked with circles and different states are labelled according to its symbolic code. Because of the symmetry all 0's may be substituted by 1's.

that, although for $\delta>1$ no Smale horseshoe exist as in the single case, the approach of orbits to $\Gamma_{0} \cup \Gamma_{1}$ (Fig. 10) is chaotic [25,28].

Figure 11 shows the value of $\delta$ (solid line) as a function of $r$ along the line $D=D_{t h}$. It is found that at $r=r_{1} \approx 22.3, \delta$ crosses one. Interested in the complex behaviours that our system could show, we focused in two values of $r$ above and below $r_{1}: r=20(\delta \approx 1.18)$ and $r=23$ $(\delta \approx 0.96)$. We do not want to carry out a detailed analysis of the solutions found, but just to show that the results have got the "flavour" expected according to previous theoretical studies on bi-homoclinicity to a saddle-focus $[25,30,31]$. 

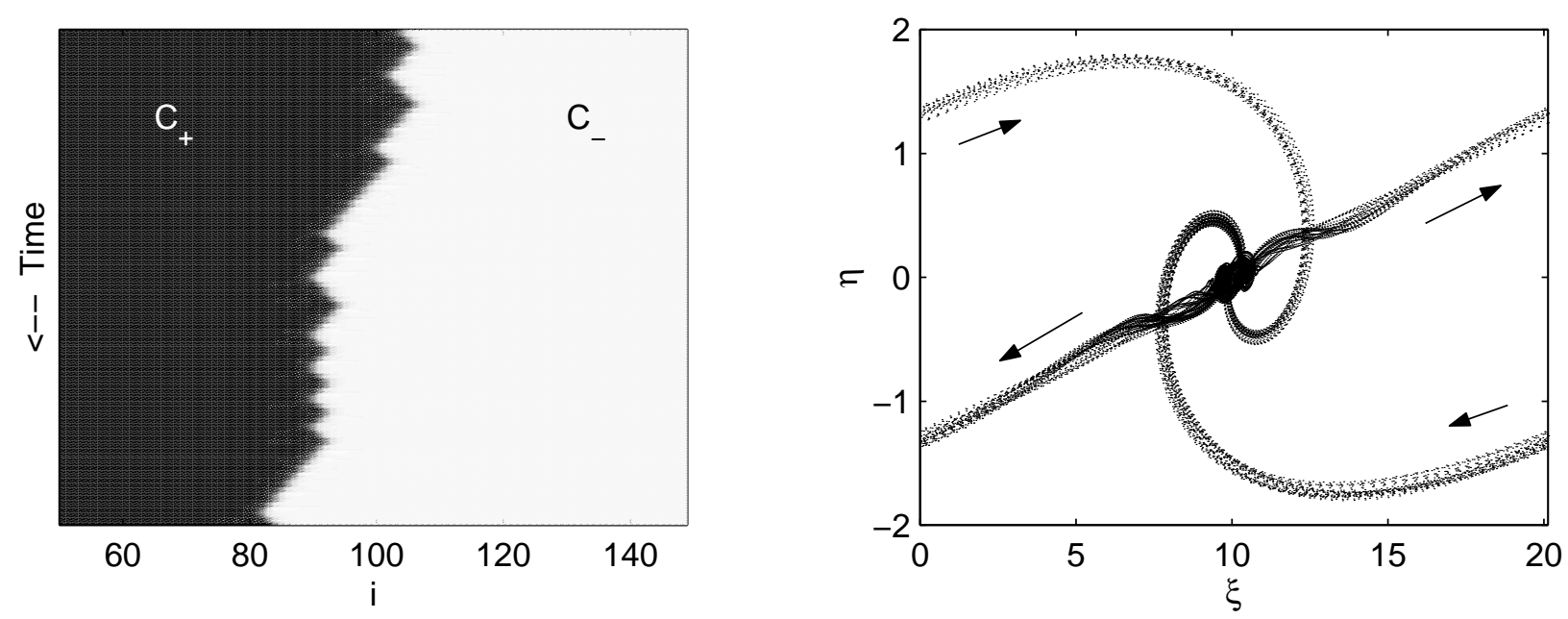

Fig. 13. Chaotic motion of the front for $r=20$ and $D=17.85$ during 200 t.u. after a transient of 30000 t.u. a) $x$ variable in gray scale where abcissa corresponds to the oscillator number and time is running downwards. b) Cylindrical variables $\xi$ and $\eta$ during the 200 t.u. shown in the left figure. The interval chosen for variable $\xi$ has been shifted half a period, with respect to previous figures, to better observe the homoclinic chaos that is organized at the $B$-state.

\section{$5.1 r=20(\delta>1)$}

In the following, we analyse the transition from a oscillating front to a travelling front for $r=20$. Fig. 12 (a) show the dependence of the period of oscillation of the front $\left(T_{1}\right)$ vs. $D$. It is found that the period does not grow continuously, and instead, there are some jumps, which give rise to hysteretic cycles (Fig. 12 (b) shows an inset of 12 (a)). These wiggles (note that unstable branches join consecutive stable branches) are not surprising according to [29], and are expected to appear when approaching $\delta=1$. Unfortunately, we have not been able to find oscillations with semi-periods larger than 3.2 t.u. This occurs because as the period grows, and therefore (bi-)homoclinicity is reached, the chaotic transients become larger and larger.

The different regimes may be labelled by their representative symbolic sequences of 0's and 1's, depending on the number of turns at each side of the saddle point. For example, the uniform propagating regimes are labeled by $\{0\}$ or $\{1\}$, but if a period-doubling occurs the new (stable) regimes are labeled by $\left\{0^{2}\right\}$ or $\left\{1^{2}\right\}$, respectively. On the other hand, the oscillating regime, described by a two-lobed cycle, is represented by the code $\{01\}$ (or $\{10\}$ ).

For the travelling front region, the dependence of the velocity with $D$ shows some features that were also reported in [29], where a system of three ordinary differential equations, that exhibits a single homoclinic connection to a saddle-focus, was studied. In Fig. 12 (c,d) the inverse of the velocity is plotted as a function of $D$. In those intervals of $D$ where no line appear, the front continues to exhibit chaotic motion, characterized by "spontaneous" front reversals, after, at least, 30000 t.u. of transient; but some small 'windows' with regular motion can be found.

Figure 13 shows the dynamics of the front for $D=17.85$ during 200 t.u., after some transient. 

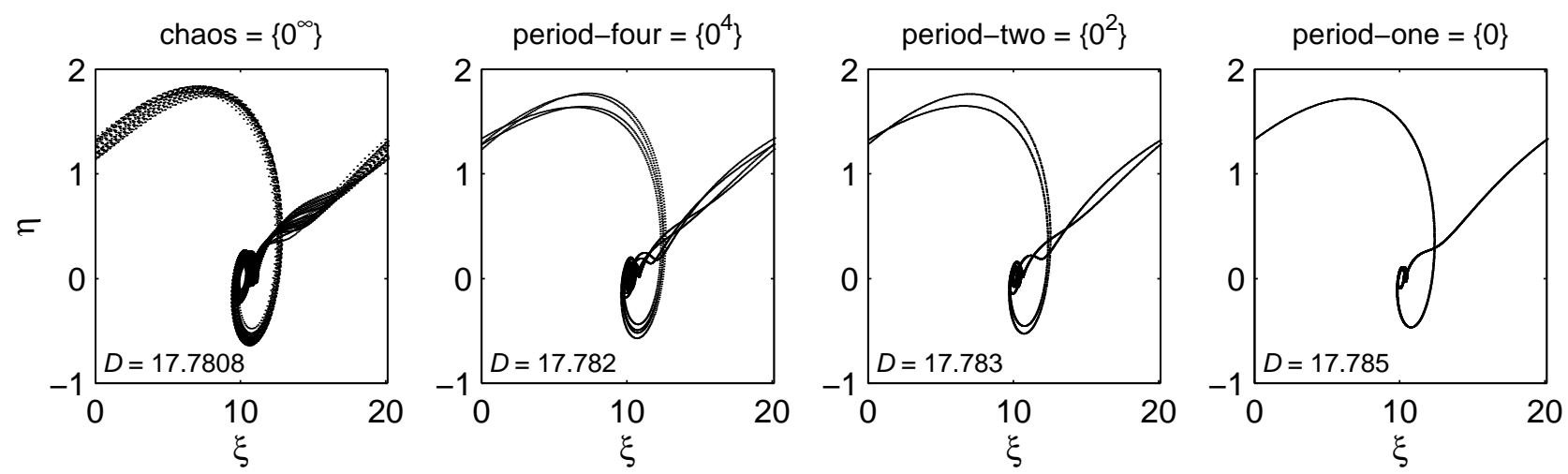

Fig. 14. From right to left a period-doubling cascade leading to chaos. Values of $D$ belong to the interval shown in the inset of Fig. 12 (c).

In Fig. 13 (a), the variable $x$ of the oscillators of the array is represented in gray scale, whereas Fig. 13 (b) shows the phase portrait $(\xi, \eta)$. The front reverses its propagation several times and displays a quasi-erratic motion. Although the behaviour is likely to be a chaotic transient, from a practical point of view, it is indistinguishable of "true chaos".

On the other hand, transitions between different regimes exhibiting sustained propagation, occur at period-doubling bifurcations (circles in Figs. $12(\mathrm{c}, \mathrm{d})$ ). In addition, we find the wellknown period-doubling route to chaos, see the inset of Fig. 12 (c). In Fig. 14 three periodic orbits and a chaotic one are shown. From right to left, a period-doubling cascade leads to a type of chaos that is characterized by sustained propagation of the front with non-periodic velocity. This scenario, that generates chaos with $\delta>1$ at homoclinicity, was already found in [29].

\section{2 $r=23(\delta<1)$}

For $r=23, \delta$ is less than one, and therefore in a small neighbourhood of $D_{t h}$, one expects to find, according to [30], Shil'nikov wiggles for both, the two-lobed cycle (oscillating front) and the one-lobed cycle (travelling front). Also we expect to find chaos of the type reported by Arneodo el al. [31]; where the strange attractor organizes around a saddle-focus with symmetry, like in Fig. 13.

However, we have seen in the previous subsection, that the local analysis is not always representative enough of the observable results that usually correspond to the lowest branch (i.e. the smallest period). In fact, our results do not differ too much from those found for $r=20$. In Fig. 15, oscillating, as well as, travelling solutions are arranged according to their periods (considering the hyper-cylindrical phase space).

The main oscillating solution (code $\{01\}$ ) disappears in a saddle-node bifurcation at $D \approx 18.04$. Surely, oscillating solutions with $\{01\}$ code and larger period exist, but we have not been able to find them. Besides the standard oscillating solution, we find others labelled $\left\{0^{2} 1^{2}\right\}$ and $\left\{0^{4} 1^{4}\right\}$ 


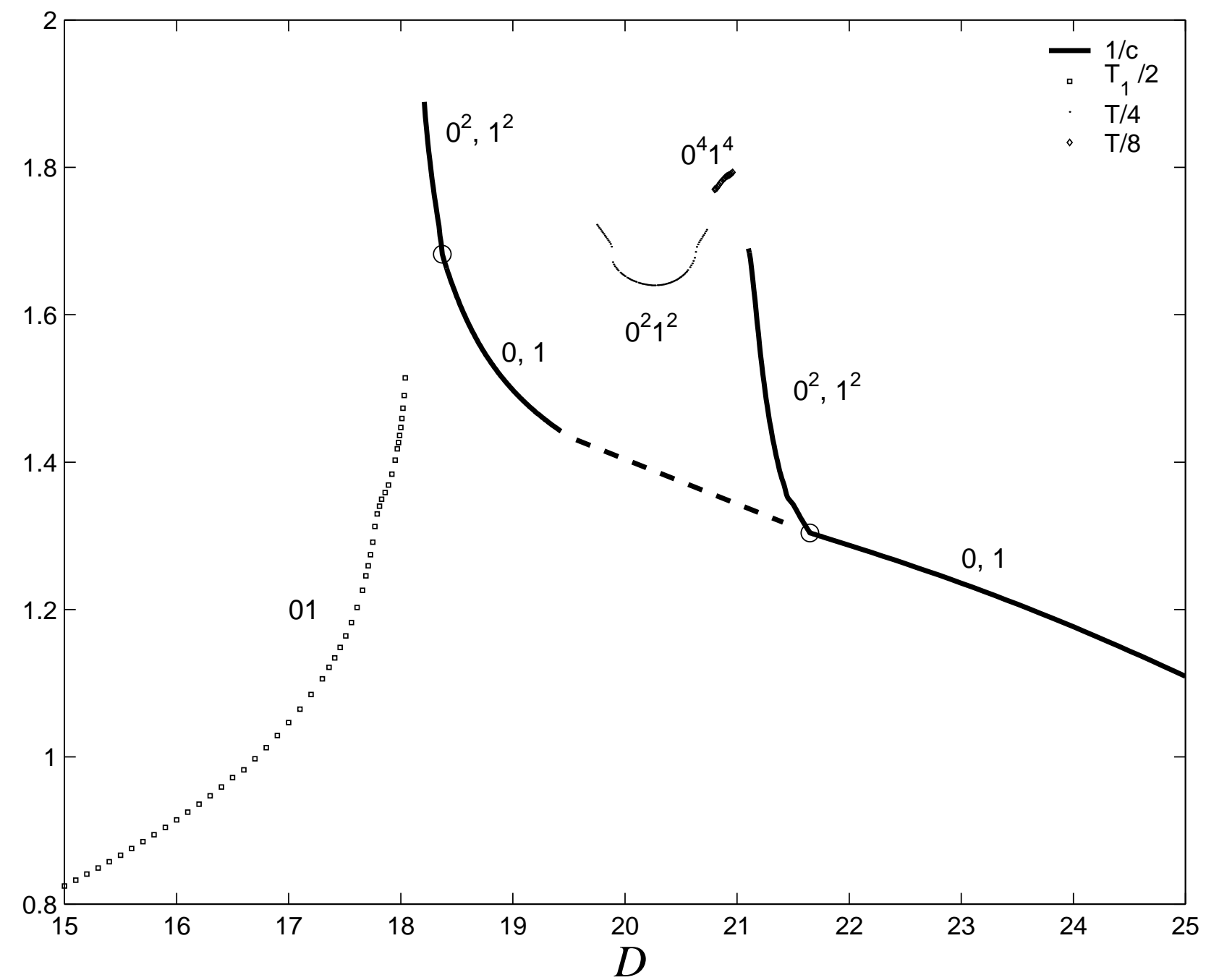

Fig. 15. $1 / c$ (solid line) and $T_{1} / 2$ (squares) as a function of $D$ for $r=23$. Two periodic states with codes $0^{2} 1^{2}$ (dots) and $0^{4} 1^{4}$ (diamonds), as well as a conjectured unstable travelling solution (dashed line) are shown.

that correspond to more sophisticated oscillating regimes. Their dynamics onto $(\xi, \eta)$ is depicted in Fig. 16. Their existence can be attributed to subsidiary homoclinic connections.

Concerning the propagating solutions (solid line in Fig. 15), the "period-one" propagating solutions (codes $\{0\}$ and $\{1\}$ ) undergo a period-doubling bifurcation at $D \approx 21.65$. Nonetheless, this "period-one" solution reappears at $D \approx 19.43$, so we conjecture, according to previous studies [30], that there exist an unstable solution (dashed line) linking both regions. The lowest values of $D$ with propagating solution exhibit another period-doubling bifurcation at $D \approx 18.37$.

For those intervals of $D$, where no periodic orbits were found, chaotic behaviour (like that of Fig. 13) is found. Nonetheless, some very small periodic windows are found intermingled into the intervals with chaotic motion. 

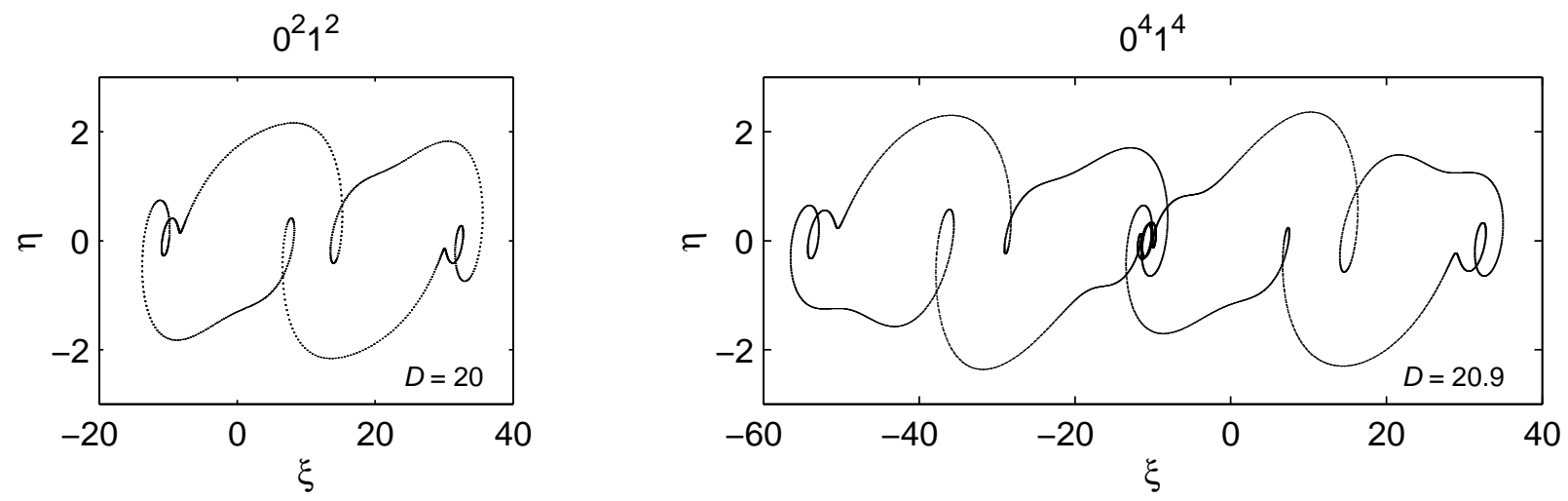

Fig. 16. Periodic orbits $0^{2} 1^{2}$ and $0^{4} 1^{4}$ for $r=23$ and two values of $D$. The cyclic restriction of the variable $\xi$ has been suppressed to better observe the periodic dynamics.

\section{$6 \quad$ Further remarks}

\subsection{Large $D$}

As the coupling $D$ is increased, a larger amount of oscillators constitute the steepest part of the front. In some sense, the front becomes more 'continuous' (or less steep). This tendency allows us to understand better the behaviour of $D_{o s}$ and $D_{t h}$ in the large $D$ region. When $D$ is large, neighbouring oscillators have similar $(x, y, z)$ values. Therefore, once the $D_{o s}$ line is crossed, a small increase of $D$ (in comparison with $D_{t h}$ or $D_{o s}$ ) is needed to achieve the multiple collision of cycles, i.e. the $D_{t h}$ line. As long as $D$ is large, both $A$ - and $B$-states are in a quasi-continuous and should exhibit similar eigenvalues spectra.

So, as $r \rightarrow r_{\infty} \approx 13.5, \lambda_{u}$ and $\lambda_{s}$ of the $B$-state at $D_{t h}$ should meet the pair of complex conjugates eigenvalues that characterize the Hopf bifurcation of the $A$-state at $D_{o s}$. In short, both lines meet at $D=\infty$ in a double-zero eigenvalue (Takens-Bogdanov) point. For this reason, oscillations above $D_{o s}$ have a small frequency and $\delta \rightarrow 1^{+}$when $r \rightarrow r_{\infty}$ (see Fig. 11).

\subsection{The effect of parameter mismatch and asymmetry}

Our system of coupled Lorenz oscillators is nothing but a mathematical model, because it is clear that it is impossible to build an experimental set-up infinitely large with identical units all of them being exactly symmetric. This means that in a real system no invariance under translation and under reflection exists.

Boundary effects can be significatively minimized by using an array sufficiently large, recall that the front is very localized, and only for large $D$ a significative amount of oscillators are located in the steepest part of the front. The effect of using non-identical units is difficult to be 
predicted but could manifest in a neighbourhood of $D_{\text {th }}$ by letting some localized oscillation to exist or making the front to reverse back at a given time. Nonetheless, there are lots of ways to make the oscillators non-identical and then giving rise to an enormous variety of effects.

More tractable is the case where all the oscillators are identical, but asymmetric. In this situation, we should use the theory of imperfect gluing bifurcations. Larger is the asymmetry, larger is the interval of $D$ at $D \approx D_{t h}$ where new periodic orbits appear. Mainly, the case when the saddle value $\tilde{\sigma}=\lambda_{u}+\operatorname{Re}\left(\lambda_{s}\right)$ is negative has been considered [33-35] showing that no more than two periodic orbits can coexist in the same parameter domain, and providing some simple rules for the symbolic sequences of 0's and 1's of the cycles, based on a Farey tree structure. Experimental studies can be found in Refs. [23] and [36]. The latter also considers the case with $\tilde{\sigma}>0$ (i.e. saddle-focus with $\delta<1$ ).

\section{$6.3 r>r_{H}$}

The behaviour of the system when the line $r=r_{H}$ is crossed, and $C_{ \pm}$become unstable, is similar to that described for the off-diagonal coupling in [32]. The transition, between oscillating and travelling fronts, becomes a transition between two well-differentiated types of spatiotemporal chaos. The slightly unstable nature of $C_{ \pm}$makes possible that domains with oscillators close to $C_{ \pm}$exist, however, sometimes one oscillator is able to jump to the other lobe, which, in the propagating region, produces the creation of two counterpropagating fronts. In short, for $D>D_{t h}$, we observe spatio-temporal chaos characterized by the spontaneous creation of counterpropagating fronts and front reversals.

\subsection{Universality?}

One may ask if the symmetry front bifurcation presented here is universal or not; in other words, in which kind of system could one expect to find this transition?

It was shown in $[18,32]$ that other coupling matrices were able to induce front propagation in an array of coupled Lorenz oscillators. If one considers coupling matrices with all elements zero except one, these two off-diagonal couplings:

$$
\begin{aligned}
& \Gamma=\gamma_{k l}=\delta_{k 1} \delta_{l 2} \\
& \Gamma=\gamma_{k l}=\delta_{k 2} \delta_{l 1}
\end{aligned}
$$

exhibit front propagation by a similar route to the one explained here. However, it may happen that the transition occurs in such a way that the standing front loses its symmetry in a small interval. This happens because a pitchfork bifurcation renders the $A$-state unstable, and when the coupling in increased further, the new (static nonsymmetric) solutions "collide" with the $B$-state transferring the stability (through a pitchfork bifurcation again). Later, it is the $B$ - 
state which undergoes a Hopf bifurcation, and finally, the oscillating solution touch the $A$-state creating the travelling solutions. The logarithmic laws, Eqs. (9) and (10), are also obtained [18].

We have also checked our results with other two bistable systems: an array whose local dynamics is a truncation of the magnetohydrodynamic partial differential equations of a disc dynamo [37], and the FitzHugh-Nagumo model [9].

The dynamo model is similar to the Lorenz system and in some parameter range it exhibits symmetric bistability. The equations of the model are:

$$
\begin{aligned}
& \dot{x}=\alpha(y-x) \\
& \dot{y}=z x-y \\
& \dot{z}=\beta-x y-\kappa z
\end{aligned}
$$

The system has one unstable fixed point at $P_{0}=(0,0, \beta / \kappa)$ and two stable fixed points $P_{ \pm}=$ $( \pm \sqrt{\beta-\kappa}, \pm \sqrt{\beta-\kappa}, 1)$ in the range $\beta \in\left(\kappa, \beta_{H}\right)$, with $\beta_{H}=15$ for $\alpha=5, \kappa=1$. We have found a transition like that with Lorenz systems, for instance for the coupling matrix $\Gamma=\gamma_{k l}=\delta_{k 2} \delta_{l 2}$. Depending on the value of $\beta$ the transition is smooth or chaotic. Whereas for $\beta=6$ we find a fine logarithmic profile of the front velocity, for $\beta=14$ the front velocity function is interrupted by a chaotic regime, like that shown in Fig. 13, when approaching the threshold. Also the oscillating dynamics, for $\beta$ close to $\beta_{H}$ is not so simple like that shown in Fig. 3.

For the discrete FitzHugh-Nagumo model:

$$
\begin{aligned}
& \dot{u}_{j}=u_{j}-u_{j}^{3}-v_{j}+D\left(u_{j+1}+u_{j-1}-2 u_{j}\right) \\
& \dot{v}_{j}=\epsilon\left(u_{j}-a_{1} v_{j}-a_{0}\right) \quad j=1, \ldots, N
\end{aligned}
$$

we take $a_{0}=0$ and $a_{1}=2$ which provide the $\mathbf{Z}_{2}$ symmetry and bistability, respectively. The local dynamics presents a saddle equilibrium point at the origin, and two odd symmetric stable fixed points $\left(u_{ \pm}, v_{ \pm}\right)=\left( \pm \sqrt{\frac{a_{1}-1}{a_{1}}}, \pm \frac{1}{a_{1}} \sqrt{\frac{a_{1}-1}{a_{1}}}\right)$. As occurs in the continuous version [16], propagation only succeeds for small $\epsilon\left(\mathcal{O}\left(10^{-1}\right)\right)$. For very small $D$, only one stable solution exits, the standing one ( $A$-state). When $D$ increases, two (counterpropagating) travelling solutions coexist with the standing one. Finally, the standing solution undergoes a subcritical Hopf bifurcation and the travelling solutions become the only stable ones. This route is apparently very different from those shown above, since a computation of the eigenvalues corresponding to the $B$-state reveals that $\lambda_{u}>-\lambda_{s}(\delta<1)$, and therefore, if any gluing bifurcation exists, it involves unstable cycles. Therefore, we speculate that the transition is as follows: When $D$ reaches a critical value $D_{S N}$, two stable travelling solutions (both propagation senses) are born with nonzero velocity $\left(c=c_{0} \neq 0\right)$, in two simultaneous saddle-node bifurcations. The unstable travelling solutions that appear in these saddle-node bifurcations become glued, when $D$ is slightly increased, at $D=D_{G}$. In this way, the (unstable) oscillating solution, that coalesces with the $A$-state at the (subcritical) Hopf bifurcation, is created.

In Fig. 17, we present the values of $1 / c$ as a function of $D$, for $\epsilon=0.1$. One may observe that 

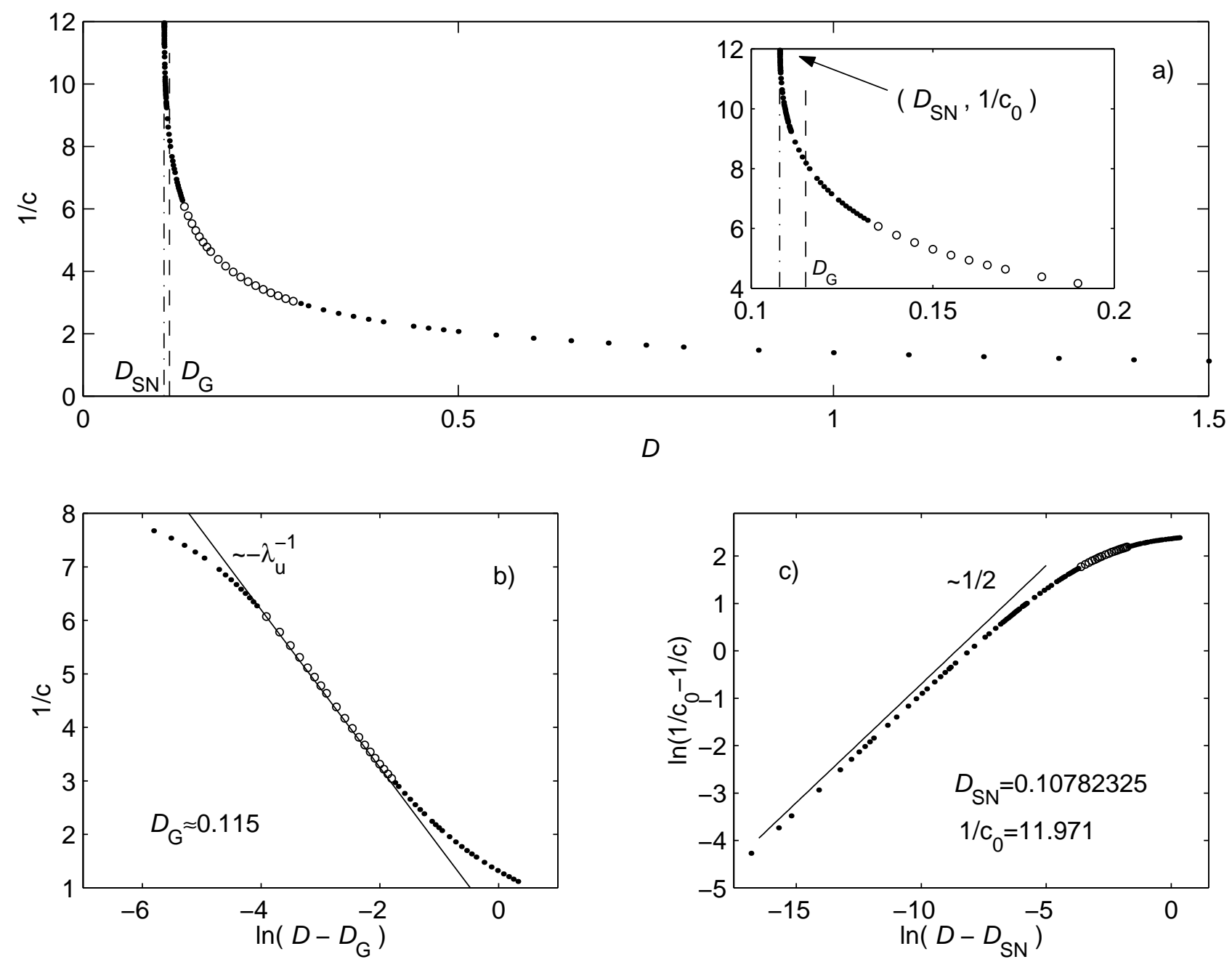

Fig. 17. Velocity of the front for the discrete FitzHugh-Nagumo model (a). Internal parameters are $a_{0}=0, a_{1}=2$, and $\epsilon=0.1$ (see Eq. (18)). Data are shown with dots and circles. It may be observed that the travelling solution ceases to exist at $D=D_{S N}$ through a saddle-node bifurcation. Nonetheless, in the interval shown with circles the increase of $1 / c$ looks logarithmical. In Fig. (b) a semilog plot shows that, at some interval, the slope of $1 / c$ agrees with the value of the unstable eigenvalue of the $B$-solution at $D_{G}: \lambda_{u}=0.6781$. This suggests that the travelling solution is approaching the (non-complex) saddle point ( $B$-state) when $D$ decreases. However the collision is prohibited, because the travelling solution is stable and the saddle index of the $B$-solution is approximately 0.7 for the values of $D$ considered, which implies that the gluing bifurcation may occur between unstable cycles only. Hence, the gluing mechanism involves the unstable travelling solutions created at $D=D_{S N}$. In Fig. (c) a log-log plot shows the dependence of $1 / c$ as a function of $D-D_{S N}$. The slope near $D_{S N}$ agrees with the expected value 0.5 , characteristic of a saddle-node bifurcation.

within a range of values of $D$ of $D, 1 / c$ exhibits a logarithmic profile (Fig. 17 (b)) but finally departs from that tendency, and shows a root-square dependence, typical of a saddle-node 
bifurcation (Fig. 17 (c)):

$$
\left(\frac{1}{c}-\frac{1}{c_{0}}\right) \propto \pm\left(D-D_{S N}\right)^{1 / 2} .
$$

In our case, the + (resp. -) sign corresponds to the unstable (resp. stable) solution. $D_{S N}$ is close to $D_{G}$ and that is the reason why the partial logarithmic dependence can be recognized in Fig. 17 (b). In fact the saddle index is not very small, $\delta \sim 0.7$, what makes possible the unstable cycles to disappear at $D_{S N}$ close to $D_{G}$.

\section{Conclusions}

It has been shown that an array composed of symmetric bistable units undergoes a front bifurcation originating two counterpropagating travelling solutions. The mechanism consists in a Hopf bifurcation of the static front, followed by a global bifurcation equivalent to a gluing bifurcation of cycles onto a cylindrical phase space. Accordingly, close to the threshold, the period of oscillation of the front $\left(T_{1}\right)$ and the speed of the front $(c)$ follow logarithmic laws. An unstable static solution mediates the gluing process, in such a way that the value of its unstable eigenvalue determines the rate of divergence of $T_{1}$ and $c^{-1}$. The transition is typically discrete, and the question of the continuum limit is intriguing.

Also, it has been demonstrated that the gluing transition may be mediated by a saddle-focus point. In that case, when the saddle index $(\delta)$ approaches one, the transition becomes much more convoluted. Different oscillating and travelling regimes are observed, included chaotic motion of the front due to Shil'nikov chaos.

In Sec. 6.4 we have dealt with other couplings and other bistable systems. We have found common features with the transition described here in detail for the Lorenz oscillator, coupled through the $y$ variable. Even in the case of the discrete FitzHugh-Nagumo model, where the transition is apparently very different, a careful examination indicates that a similar scenario exists, despite the gluing bifurcation is "invisible".

A further investigation is needed to know a priori what local dynamics and what couplings are suitable to achieve propagation in a discrete symmetric bistable medium. Besides the multivariable nature of the local dynamics, some trivial considerations about the coupling, and the observation that foci are more adequate than sinks, additional arguments are still to be developed. We hope that future research will clarify this point.

\section{Acknowledgments}

The support by MCyT under Research Grant BFM2000-0348 is gratefully acknowledged. 


\section{References}

[1] A.M. Turing, Philos. Trans. R. Soc. London B327, 37 (1952).

[2] P.C. Fife, Mathematical Aspects of Reacting and Diffusing Systems (Springer-Verlag, New York, 1979).

[3] J.D. Murray, Mathematical Biology (Springer-Verlag, New York, 1993).

[4] M.C. Cross and P.C. Hohenberg, Rev. Mod. Phys. 65, 851 (1993). C. Bowman and A.C. Newell, ibid. 70, 289 (1998).

[5] Optical Bistability; edited by C.M. Bowden, M. Ciftan and J.R. Robl (Plenum, New York, 1981).

[6] W.J. Firth, Phys. Rev. Lett. 61, 329 (1988).

[7] J.P. Laplante and T. Erneux, J. Phys. Chem. 96, 4931 (1992).

[8] H.P. McKean, Adv. in Math. 4, 209 (1970).

[9] R. FitzHugh, Biophys. J. 1, 445 (1961).

[10] J.P. Keener, SIAM J. Appl. Math. 47, 556 (1987).

[11] T. Erneux and G. Nicolis, Physica D 67, 237 (1993).

[12] V. Pérez-Muñuzuri, V. Pérez-Villar and L. O. Chua, Int. J. Bif. and Chaos 2, 403 (1992); A.P. Muñuzuri, V. Pérez-Muñuzuri, M. Gómez-Gesteira, L.O. Chua and V. Pérez-Villar, ibid. 5, 17 (1995).

[13] R.S. MacKay and J.-A. Sepulchre, Physica D 82, 243 (1995).

[14] K. Kladko, I. Mitkov and A.R. Bishop, Phys. Rev. Lett. 84, 4505 (2000).

[15] A. Carpio and L.L. Bonilla, Phys. Rev. Lett. 86, 6034 (2001).

[16] A. Hagberg and E. Meron, Nonlinearity 7, 805 (1994); Chaos 4, 477 (1994).

[17] P. Coullet, J. Lega, B. Houchmanzadeh and J. Lajzerowicz, Phys. Rev. Lett. 65, 1352 (1990).

[18] D. Pazó and V. Pérez-Muñuzuri, Phys. Rev. E 64, 065203(R) (2001).

[19] G. Fáth, Physica D 116, 176 (1998).

[20] E.N. Lorenz, J. Atmos. Sci. 20, 130 (1963). H. Haken, Phys. Lett. A 53, 71 (1975).

[21] C. Sparrow, The Lorenz Equations: Bifurcations, Chaos and Strange Attractors (Springer-Verlag, New York, 1982).

[22] J.M. Lopez and F. Marques, Phys. Rev. Lett. 85, 972 (2000); F. Marques, J.M. Lopez, J. Shen, Physica D 156, 81 (2001).

[23] J.I. Rosell, J. Farjas, R. Herrero, F. Pi and G. Orriols, Physica D 85, 509 (1995); R. Herrero, J. Farjas, R. Pons, F. Pi and G. Orriols, Phys. Rev. E 57, 5366 (1998). 
[24] S.H. Strogatz, Nonlinear dynamics and chaos: with applications to physics, biology, chemistry, and engineering (Perseus Books, Reading, 1994). P. Glendinning, Stability, instability and chaos: an introduction to the theory of nonlinear differential equations (Cambridge Univerity Press, 1994).

[25] P.J. Holmes, J. Diff. Eq. 37, 382 (1980).

[26] L.P. Shil'nikov, Sov. Math. Dokl. 6, 163 (1965).

[27] J. Guckenheimer and P. Holmes, Nonlinear Oscillations, Dynamical Systems, and Bifurcations of Vector Fields (Springer-Verlag, New York, 1983)

[28] S. Wiggins, Global Bifurcations and Chaos (Springer-Verlag, New York, 1988).

[29] P. Glendinning and C. Sparrow, J. Stat. Phys. 35, 645 (1984).

[30] P. Glendinning, Phys. Lett. A 103, 163 (1984).

[31] A. Arneodo, P. Coullet and C. Tresser, Commun. Math. Phys. 79, 573 (1981).

[32] D. Pazó, N. Montejo and V. Pérez-Muñuzuri, Phys. Rev. E 63, 066206 (2001).

[33] D.V. Turaev and L.P. Shil'nikov, Sov. Math. Dokl. 34, 397 (1987).

[34] J.M. Gambaudo, P. Glendinning and C. Tresser, in Instabilities and Nonequilibrium Structures, edited by E. Tirapegui and D. Villarroel (D. Reidel, Dordrecht, 1987).

[35] J.M. Gambaudo, P. Glendinning and C. Tresser, Nonlinearity 1, 203 (1988).

[36] P. Glendinning, J. Abshagen and T. Mullin, Phys. Rev. E 64, 036208 (2001).

[37] D.R.J. Chillingwoths and R.J. Holmes, Math. Geol. 12, 41 (1980). 